\title{
Determination of concentric and eccentric peak moment values for trunk flexion and extension in sedentary asymptomatic individuals by isokinetic dynamometry: a pilot study
}

\author{
Rafaella Stradiotto Bernardelli ${ }^{*}$, Auristela Duarte de Lima Moser ${ }^{1}$, Gerson Linck Bichinho ${ }^{1}$ \\ ORIGINAL ARTICLE
}

\begin{abstract}
The spine has a direct influence on postural alignment and movement of the whole body. Lumbar muscles constitute a critical element in trunk performance while weakness of these muscles has been associated with low back pain. Hence, strength profiling of trunk muscles is clinically significant. The objective of this research was to determine, by means of isokinetic dynamometry, peak moment (PM) values during isokinetic concentric and eccentric efforts of trunk flexion and extension in sedentary asymptomatic individuals. The sample consisted of 100 asymptomatic sedentary volunteers, fifty from each sex, aging $22.2 \pm 3.3$ years old. The sample underwent concentric and eccentric isokinetic assessment of the trunk flexor and extensor muscles at an angular velocity of 60 degrees/sec for each mode of contraction. The mean concentric PM for trunk flexion and extension were 139.5 and $166.6 \mathrm{Nm}$, respectively, while the respective values for the eccentric efforts were 188.8 and $221.2 \mathrm{Nm}$. The PM flexion/extension ratio was 0.87 and 0.89 for the concentric and eccentric efforts, respectively. These values of concentric and eccentric PM and PM ratio will serve as comparison parameters for future research, as well as for the assessment of symptomatic patients, and to help in the creation of the trunk muscle rebalance protocols.

Keywords: Skeletal muscle; Spine; Muscle strength; Dynamometer.
\end{abstract}

\section{INTRODUCTION}

The spine forms a closed kinematic chain through segmental coordination of the upper and lower extremities and the pelvic girdle, and affects the static and dynamic patterns of daily life activities (Jeon \& Kim, 2016; Pinto et al., 2008), while also having a direct influence on postural alignment and movement of the whole body through musculoskeletal connections (Haight, Dahm, Smith, \& Krause, 2005; Jeon \& Kim, 2016).

The trunk musculature plays a fundamental role when it is required to act against imbalances imposed on the joints. Several studies and reviews show that muscle strength and balance are primary factors in lumbar stability (Hides, Stanton, Mendis, \& Sexton, 2011; Lee et al., 1999; Silva Neto, Simões, Grangeiro Neto, \& Cardone, 2010; van Middelkoop et al., 2011; van Middelkoop et al., 2010).
Data from the World Health Organization show that about $80-85 \%$ of back pain episodes worldwide have no known cause. Low back pain (LBP) is the most common spine disorder, affecting more than $80 \%$ of people at some point in their lives, and $90 \%$ of those affected have more than one episode. Low back pain has been identified as a frequent cause of disability among young adults (WHO, 2013).

Thus, there has been an increase in research related to methods of treatment, prevention, and diagnosis of lumbar dysfunction. Most of these studies have assessed the relationship between trunk muscle strength and LBP and show weakness and imbalance of these muscles in individuals with this and other spine disorder (Lim, Poh, Low, \& Wong, 2011; Marshall, Kennedy, Brooks, \& Lonsdale, 2013; Mostagi et al., 2015; Pereira et al., 2012; Wajswelner, Metcalf, \& Bennell, 2012; Wells, Kolt, Marshall,

\footnotetext{
Manuscript received at June $1^{\text {st }} 2016$; Accepted at February $2^{\text {nd }} 2017$

${ }^{1}$ Pontifical Catholic University of Parana - PUCPR

* Corresponding author: Pontifical Catholic University of Parana - PUCPR, Curitba-Brazil. E-mail: rafaellasb@yahoo.com.br
} 
Hill, \& Bialocerkowski, 2013; Yahia et al., 2010; Yahia et al., 2011).

The development of modern dynamometers allows better measurement of muscle parameters such as torque production capacity, muscle power, fatigue, and work in isokinetic contraction. These dynamometers also enable recording of maximum muscle strength in all ranges of motion. Isokinetic dynamometry is one of the most widely used objective methods to test trunk muscle strength, and more recently, it has been used in strength training. (Grabiner, Jeziorowski, \& Divekar, 1990; Newton \& Waddell, 1993). It measures trunk flexion and extension strength at various angular speeds and contraction modes (isometric, concentric, and eccentric), and has been found to be safe (den Hartog et al., 2010), reliable (Hupli, Sainio, Hurri, \& Alaranta, 1997), validated (Guilhem, Giroux, Couturier, \& Maffiuletti, 2014), and sensitive enough to detect muscle weakness (Barczyk-Pawelec, Piechura, Dziubek, \& Rozek, 2015; Cho et al., 2014).

There is great benefit in using trunk isokinetic dynamometry to reliably assess strength parameters (Barczyk-Pawelec et al., 2015; Guilhem et al., 2014; Jeon \& Kim, 2016; Newton \& Waddell, 1993). There is a lack of normative data of strength of flexors and extensors trunk muscles in the literature. In especial, the lack of normative data from asymptomatic sedentary adults hinders the accurate assessment of muscle parameters in other populations, for example, individuals with low back disorders and athletes. This is because, unlike of the arms and legs that may have the strength of a limb compared to the contralateral limb, the trunk does not present this possibility. In this way, the comparison of the trunk strength of an individual always will need to compare with normative data or parameters of normality. Therefore, this study may contribute to the establishment of normal parameters for future studies with larger samples in this population.

Hence, this study aimed to use isokinetic dynamometry to determine peak moment (PM) values in concentric and eccentric contractions in trunk flexion and extension, and the relationship between these values in asymptomatic sedentary subjects.

\section{METHOD}

This research is an evaluative, cross-sectional, exploratory, descriptive, experimental pilot study. Experiments were carried out at the Isokinetic Dynamometry Center of the Physiotherapy Clinic at Pontifical Catholic University of Paraná - Brazil, after approval by the Human Research Ethics Committee of the same institution, under protocol number: 249771.

\section{Participants}

The participants consisted of 100 sedentary individuals asymptomatic for LBP, 50 of each sex, aged 18-30 years old, who agreed to participate in the research by providing a signed and informed consent before enrolment. The inclusion criterion was that individuals were classified as sedentary, that is, not practicing regular physical activity (less than 150 minutes of vigorous-intensity physical activity throughout the week, or an equivalent combination of moderate and vigorous intensity activity, or muscle-strengthening activities less than twice a week (WHO, 2010)). Individuals with evident postural change, spine or hip osteomyoarticular lesions, abdominal or spine surgeries, pain in the lumbar region, knee injuries and misalignments, and pregnant women were excluded. Individuals who failed to correctly complete the test, i.e., who stopped or reversed force direction during the test, were excluded. The participants consisted of PUCPR students, who were invited by the researchers to participate in this project.

\section{Measures and Procedures}

Each participant underwent an isokinetic assessment following a protocol that tested the trunk flexor and extensor musculature in concentric and eccentric contraction, starting with $10^{\circ}$ of extension and going up to $70^{\circ}$ of flexion, with five repetitions at an angular velocity of $60 \%$ s (den Hartog et al., 2010; Gómez et al., 2005; Hupli et al., 1997; Silva Neto et al., 2010; Yahia et al., 2010; Yahia et al., 2011). The protocol is described in Figure 1. 


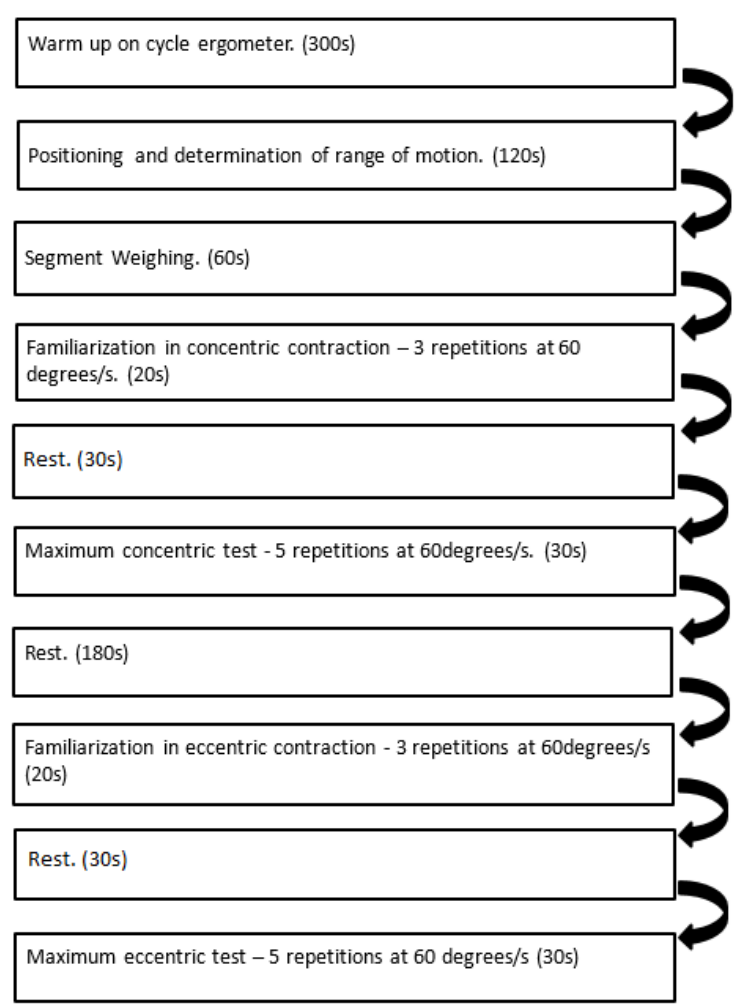

Figure 1. Scheme describing the methodological procedures of isokinetic evaluation, with approximate duration of each step.

Participants were positioned on the dynamometer in accordance with the technical standards guide of the equipment as prescribed by the manufacturer (Cybex ${ }^{\circledR}$, model NORM 700011) (CYBEX, 1995) in the specific module for analysing trunk strength. The participant remained in the same position throughout the test.

Each volunteer was placed standing with semi-flexed knees, limited behind in the popliteal line, and below and above the patella in front; the drive shaft of the dynamometer was positioned at the L4/L5 level, with a fastening belt at the hip, and with the shoulder girdle region fixed by a belt above the nipples; only free flexion and extension of the trunk was possible (CYBEX, 1995).

After proper attachment of the individual to the equipment, the trunk was weighed by the device in a $45^{\circ}$ flexion position, and this value was subtracted from the body weight (CYBEX, 1995). The same researcher positioned and attached all subjects in this experiment to avoid bias.

During the tests, all subjects received the same verbal stimulus in order to develop maximum strength. The selected parameter for

muscle performance assessment was peak moment, in Newton meters (Nm).

\section{Statistical Analysis}

Results were expressed as mean, median, minimum and maximum values, and standard deviations. Pearson's correlation coefficient was estimated to establish the relationship between the parameters. For comparisons between groups, Student's t-test was considered for independent as well as paired samples. The analysis of covariance (ANCOVA) was used for comparisons between groups, keeping another quantitative covariable, and considering a statistical significance of $5 \%$.

\section{RESULTS}

The mean age of participants was $22.2 \pm 3.3$ years, and the body mass index (BMI) was $24.4 \pm$ $4.0 \mathrm{~kg} / \mathrm{cm}^{2}$ (Table 1).

Table 1

Comparison of age and BMI between sexes

\begin{tabular}{cccc}
\hline Variable & Sex & $\begin{array}{c}\text { Mean } \pm \text { Standard } \\
\text { deviation }\end{array}$ & $\begin{array}{c}P \\
\text { value }\end{array}$ \\
\hline \multirow{2}{*}{ Age (years) } & Male & $22.74 \pm 3.70$ & \multirow{2}{*}{0.116} \\
\cline { 2 - 3 } & Female & $21.68 \pm 2.93$ & \\
\hline \multirow{2}{*}{ BMI $\left(\mathrm{Kg} / \mathrm{cm}^{2}\right)$} & Male & $24.95 \pm 4.12$ & \multirow{2}{*}{0.196} \\
\cline { 2 - 3 } & Female & $23.90 \pm 3.93$ & \\
\hline
\end{tabular}

Significance of Student's t-test for independent samples, $p<0.05$. BMI: Body Mass Index.

Table 2

Correlation between BMI and isokinetic variables

\begin{tabular}{|c|c|c|}
\hline Variable & $\begin{array}{l}\text { Correlation } \\
\text { Coefficient }\end{array}$ & $P$ value \\
\hline BMI X Flexion CON PM & 0.408 & $<0.00$ \\
\hline BMI X Flexion CON PM angle & 0.072 & 0.477 \\
\hline BMI X Extension CON PM & 0.261 & 0.009 \\
\hline BMI X Extension CON PM ar & -0.008 & 0.935 \\
\hline BMI X Flex/Ext CON ratio & 0.119 & 0.239 \\
\hline BMI X Flexion ECC PM & 0.379 & $<0.001$ \\
\hline BMI X Flexion ECC PM an & -0.322 & 0.001 \\
\hline BMI X Extension ECC PM & 0.311 & 0.002 \\
\hline BMI X Extension ECC PM a & 0.067 & 0.508 \\
\hline BMI X Flex/Ext ECC ratio & 0.001 & 0.991 \\
\hline \multicolumn{3}{|c|}{$\begin{array}{l}\text { Significance of Pearson's correlation coefficient, } p<0.05 \text {. BMI: } \\
\text { Body Mass Index; CON PM: concentric peak moment; ECC } \\
\text { PM: eccentric peak moment; Flex/Ext CON ratio: relationship } \\
\text { between flexion concentric peak moment and extension } \\
\text { concentric peak moment; Flex/Ext ECC ratio: relationship } \\
\text { between flexion eccentric peak moment and extension } \\
\text { eccentric peak moment. }\end{array}$} \\
\hline
\end{tabular}


The evaluation of the correlations between BMI and the variables analysed with isokinetic dynamometry are described in Table 2 .

Although no significant differences were found for BMI when stratified according to sex (Table 1), Table 2 shows that BMI had a significant correlation with the following isokinetic variables: trunk flexion concentric peak moment (Flexion CON PM); trunk extension concentric peak moment (Extension CON PM); trunk flexion eccentric peak moment (Flexion ECC PM); trunk flexion eccentric peak moment angle (Flexion ECC PM angle); and trunk extension eccentric peak moment (Extension ECC PM). This result shows that $50 \%$ of the isokinetic data have a significant correlation with BMI. For this reason, it was decided to perform the analysis of the isokinetic data stratified according to sex set to BMI.

The concentric and eccentric PM values obtained are shown in Table 3, for total sample and stratified according to sex. The results of the comparison between these variables when stratified according to sex are Also shown.

The results in Table 3 show a significant difference for the variables of concentric PM for both trunk flexor $(p<0.001)$ and extensor muscles $(p<0.001)$, when comparing sexes. Men showed higher mean values for both flexor and extensor muscle concentric PM.

Table 3

Descriptive statistics of each concentric and eccentric isokinetic variable and the $p$ values from the statistical tests

\begin{tabular}{|c|c|c|c|c|c|}
\hline Variable & Sex & $\mathrm{N}$ & $\begin{array}{c}\text { Mean } \pm \text { Standard } \\
\text { deviation }\end{array}$ & $\begin{array}{c}\text { Median } \\
\text { (Min. - Max.) }\end{array}$ & $p$ value \\
\hline \multirow{3}{*}{ Flexion CON PM (N/m) } & $\mathrm{M}$ & 50 & $168.72 \pm 26.58$ & $166.5(110-229)$ & \multirow{3}{*}{$<0.001$} \\
\hline & $\mathrm{F}$ & 50 & $110.38 \pm 22.75$ & $108.5(71-170)$ & \\
\hline & Total & 100 & $139.55 \pm 38.28$ & $137.0(71-229)$ & \\
\hline \multirow{3}{*}{ Flexion CON PM Angle (degrees) } & M & 50 & $55.22 \pm 8.37$ & $58.5(32-65)$ & \multirow{3}{*}{0.103} \\
\hline & $\mathrm{F}$ & 50 & $57.42 \pm 5.65$ & $58.0(46-65)$ & \\
\hline & Total & 100 & $56.32 \pm 7.19$ & $58.0(32-65)$ & \\
\hline \multirow{3}{*}{ Extension CON PM (N/m) } & $\mathrm{M}$ & 50 & $210.26 \pm 37.99$ & $207.0(141-300)$ & \multirow{3}{*}{$<0.001$} \\
\hline & $\mathrm{F}$ & 50 & $123.06 \pm 30.63$ & $117.0(81-250)$ & \\
\hline & Total & 100 & $166.66 \pm 55.66$ & $161.5(81-300)$ & \\
\hline \multirow{3}{*}{ Extension CON PM Angle (degrees) } & M & 50 & $41.54 \pm 6.45$ & $42.5(27-52)$ & \multirow{3}{*}{0.044} \\
\hline & $\mathrm{F}$ & 50 & $38.26 \pm 9.46$ & $39.0(5-53)$ & \\
\hline & Total & 100 & $39.90 \pm 8.22$ & $41.0(5-53)$ & \\
\hline \multirow{3}{*}{ Ext/Flex CON Ratio (\%) } & $\mathrm{M}$ & 50 & $81.42 \pm 12.14$ & $81.0(54-114)$ & \multirow{3}{*}{0.002} \\
\hline & $\mathrm{F}$ & 50 & $92.74 \pm 23.31$ & $84.5(66-172)$ & \\
\hline & Total & 100 & $87.08 \pm 19.35$ & $84.0(54-172)$ & \\
\hline \multirow{3}{*}{ Flexion ECC PM (N/m) } & M & 50 & $227.64 \pm 33.35$ & $230.5(165-297)$ & \multirow{3}{*}{$<0.001$} \\
\hline & $\mathrm{F}$ & 50 & $150.04 \pm 24.14$ & $149.0(99-210)$ & \\
\hline & Total & 100 & $188.84 \pm 48.57$ & $180.0(99-297)$ & \\
\hline \multirow{3}{*}{ Flexion ECC PM Angle (degrees) } & $\mathrm{M}$ & 50 & $51.24 \pm 6.10$ & $53.0(30-61)$ & \multirow{3}{*}{0.005} \\
\hline & $\mathrm{F}$ & 50 & $54.88 \pm 5.40$ & $56.0(39-65)$ & \\
\hline & Total & 100 & $53.06 \pm 6.02$ & $54.0(30-65)$ & \\
\hline \multirow{3}{*}{ Extension ECC PM (N/m) } & $\mathrm{M}$ & 50 & $283.24 \pm 57.49$ & $287.0(179-397)$ & \multirow{3}{*}{$<0.001$} \\
\hline & $\mathrm{F}$ & 50 & $159.14 \pm 30.68$ & $155.5(108-220)$ & \\
\hline & Total & 100 & $221.19 \pm 77.40$ & $209.0(108-397)$ & \\
\hline \multirow{3}{*}{ Extension ECC PM Angle (degrees) } & M & 50 & $37.58 \pm 12.60$ & $36.0(14-62)$ & \multirow{3}{*}{0.023} \\
\hline & $\mathrm{F}$ & 50 & $30.26 \pm 17.53$ & $29.0(5-67)$ & \\
\hline & Total & 100 & $33.92 \pm 15.63$ & $33.0(5-67)$ & \\
\hline \multirow{3}{*}{ Ext/Flex ECC Ratio (\%) } & $\mathrm{M}$ & 50 & $82.26 \pm 12.98$ & $82.0(58-121)$ & \multirow{3}{*}{$<0.001$} \\
\hline & F & 50 & $96.54 \pm 18.78$ & $92.0(65-142)$ & \\
\hline & Total & 100 & $89.40 \pm 17.59$ & $87.5(58-142)$ & \\
\hline
\end{tabular}

Significance of ANCOVA model, $p<0.05$. CON PT: concentric peak moment; Flex/Ext CON ratio: relationship between flexion concentric peak moment and extension concentric peak moment; ECC PM: eccentric peak moment; Flex/Ext ECC ratio: relationship between flexion eccentric peak moment and extension eccentric peak moment; Nm: Newton meters; \%: percent.

The variables referring to the concentric relationship between trunk extensor and flexor muscles $(p=0.002)$ showed higher mean values for women. The trunk extension concentric PM 
angle also differed between sexes $(p=0.044)$, and was higher (occurring when the trunk was more flexed) for men. On the other hand, the trunk flexion concentric PM angle did not present significantly different mean values between sexes $(p=0.103)$. Similar to the concentric isokinetic variables, the results of the eccentric peak moment variables also show a significant difference when comparing sexes, for both the trunk flexor $(p<0.001)$ and extensor muscles $(p<0.001)$, with higher PM mean values for men.

Mean values for angles also showed significant differences between sexes. The eccentric PM angle of trunk flexors was higher in women $(p=0.005)$, whereas the eccentric PM angle of trunk extensors was higher in men $(p=0.023)$.

The eccentric relationship between trunk extensor and flexor muscles showed a significantly higher mean for women $(p<0.001)$. When compare concentric and eccentric PM of the sample, globally and stratified according to sex, the results of the Student's t-test show a significant difference when comparing the values of concentric PM with those of eccentric PM for trunk flexor and extensor muscles. The eccentric PM mean value was higher than the concentric value $(p=0.001)$ in both sexes and in total sample for flexors and extensors.

Table 4

Evaluation of the correlation between concentric and eccentric peak moment

\begin{tabular}{ccccc}
\hline Variable & Sex & $\mathrm{N}$ & Correlation Coefficient & $P$ value \\
\hline Flexion CON PM & $\mathrm{M}$ & 50 & 0.670 & $<0.000$ \\
\cline { 2 - 5 }$\times$ & $\mathrm{F}$ & 50 & 0.524 & $<0.000$ \\
\cline { 2 - 5 } Flexion ECC PM & Total & 100 & 0.850 & $<0.000$ \\
\hline Extension CON PM & $\mathrm{M}$ & 50 & 0.537 & $<0.000$ \\
$\times$ & $\mathrm{F}$ & 50 & 0.482 & $<0.000$ \\
\cline { 2 - 5 } Extension ECC PM & Total & 100 & 0.821 & $<0.000$ \\
\hline
\end{tabular}

Significance of Pearson's correlation coefficient, $\mathrm{p}<0.05$. CON PM: concentric peak moment; ECC PM: eccentric peak moment.

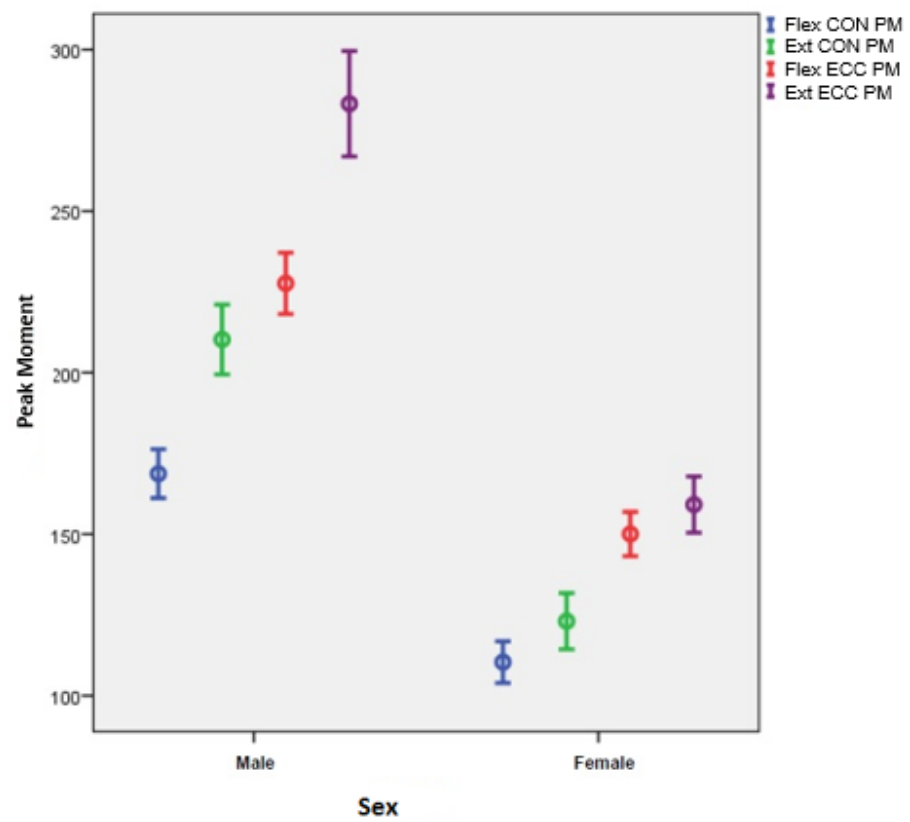

Figure 2. Comparison between the variables of peak moment stratified according to sex. The figure shows the mean and standard deviation values of the peak moment variables according to sex. Flex CON PM = flexion concentric peak moment; Ext CON PM = extension concentric peak moment; Flex ECC PM = flexion eccentric peak moment; Ext CON PM = extension eccentric peak moment.

The results of the evaluation of the correlation between concentric and eccentric PM of the sample, globally and stratified according to sex (table 4) show a significant positive correlation between the values of concentric and eccentric PM of trunk flexor muscles as well as between the values of concentric and eccentric PM of 
trunk extensor muscles, for both the total and sex-stratified sample.

It was then observed that the individuals' concentric and eccentric PM values were directly proportional and correlated with one another, although they had significantly different mean values, as shown in figure 2. Figure 2 also shows the results for peak moment presented in the previous tables. It was observed a higher PM values for male subjects compared with female subjects, both in concentric and eccentric contraction of flexor and extensor muscles.

\section{DISCUSSION}

It is known that normative data or common values are important to characterize populations, to identify parameters of normality and variations of these and to compare the characteristics between distinct populations. This statement is true also for parameters of strength. It is known that muscular strength varies according to population characteristics such as age, sex, level of physical activity, among others. The isokinetic dynamometer is an accurate equipment, capturing small variations in muscle strength parameters, as in the case of PM in this study. Therefore, it is important to clarify that when comparing PM values between different studies, it is important to consider the characteristics of the population and the characteristics of the isokinetic dynamometer, as well as the parameters, such as speed, number of repetition and mode of contraction, stipulated for the isokinetic test.

Delitto, Rose, Crandell, and Strube (1991) assessed 29 asymptomatic men and 32 asymptomatic women with a mean age of 40 years old. In this study, three trunk isokinetic tests were performed at an angular velocity of $60 \%$ in concentric mode, with a one-week interval between the first and second testing, and two weeks between the second and third. The data presented the mean values of the three assessments. The peak moment mean was $58 \mathrm{Nm}$ for flexor muscles and $82 \mathrm{Nm}$ for extensors in women. In men, the values were $81 \mathrm{Nm}$ for flexors and $111 \mathrm{Nm}$ for extensors (Delitto, Rose, Crandell, \& Strube, 1991).
It is observed that the current study presents values of concentric peak moment higher than those found by Delitto et al. (1991). An accurate comparison of these values between studies is hampered by the lack of data on range of motion and age groups of the individuals. However, the current findings corroborate the previous results regarding the higher mean force found for the extensor muscles compared with the flexors, as well as for the higher peak moment mean values found in men.

Gómez et al. (2005) performed an isokinetic evaluation of trunk muscle concentric contraction in 38 females and 35 male volunteers, aged 20 to 39 years old, testing angular velocity of $60^{\circ} / \mathrm{s}$ and range of motion of $70^{\circ}$ of flexion and $10^{\circ}$ of extension. The PM mean was $204 \mathrm{Nm}$ for flexors and $235 \mathrm{Nm}$ for extensors in women. In men, the mean values were $284 \mathrm{Nm}$ for the flexors and $360 \mathrm{Nm}$ for the extensors (Gómez et al., 2005). The peak moment values shown in Table 3 are lower than those found by Gómez, but the study did not specify whether the individuals had any regular physical activity, which could explain the higher values. It is worth noting that the study by Gómez et al. (2005) has a smaller sample than our study and like that of Delitto et al. (1991), only evaluated the participants in concentric contraction.

Yahia et al. (2010) compared the strength of individuals with chronic sciatic pain with asymptomatic individuals. Of 68 individuals of both sexes that were assessed, 40 had no lumbar pain, while the other 28 had chronic sciatic pain symptoms. Concentric testing was performed with an angular velocity of $60^{\circ} / \mathrm{sec}$ and a range of motion of $70^{\circ}$ of flexion and $0^{\circ}$ of extension. Asymptomatic subjects showed $177.44 \mathrm{Nm}$ and 192.73 Nm for flexion and extension peak moment mean, respectively. On the other hand, symptomatic subjects showed flexion peak moment mean values of $134.32 \mathrm{Nm}$ and extension peak moment mean values of 123.71 Nm (Yahia et al., 2010).

In order to investigate postural characteristics of the trunk and lower limbs, as well as strength profiles in subjects with chronic LBP, Yahia et al. (2011) assessed 60 individuals, predominantly 
women; 30 had LBP (mean age 41.1 years old), and 30 were asymptomatic (mean age 39 years old). Trunk concentric isokinetic testing was performed with an angular velocity of $60^{\circ} / \mathrm{sec}$, and range of motion of $70^{\circ}$ of flexion and $0^{\circ}$ of extension. For asymptomatic individuals, the flexion peak moment mean was $122.5 \mathrm{Nm}$ and extension peak moment mean was $144.8 \mathrm{Nm}$. For symptomatic individuals, the flexion and extension peak moment means were $102.06 \mathrm{Nm}$ and $75.7 \mathrm{Nm}$, respectively (Yahia et al., 2011). The results for the asymptomatic group of the study by Yahia et al. (2011) are similar to those shown in Table 3, while the asymptomatic group of the study by Yahia et al. (2010) have higher peak moment values than those of our study.

The present study showed that women have significantly lower peak moment values than men in trunk extension and flexion in concentric and eccentric contraction (Table 3), as shown by Van Damme et al. (2013) in assessing healthy individuals at angular velocities of $30 \% \mathrm{~s}, 60 \% \mathrm{~s}$, $90 \%$ s, and $120 \%$ s in concentric contraction.

In a validation study of isokinetic trunk dynamometry by Guilhem, Giroux, Couturier, and Maffiuletti, (2014), 15 healthy young adults were evaluated, and PM values were higher than those obtained in the current study for both concentric and eccentric contraction. However, it is not known whether these subjects were sedentary or not. Their data showed that eccentric peak moment values are higher than concentric values (Guilhem et al., 2014), which corroborates our results. The results show significant differences when comparing peak moment values for concentric and eccentric of trunk flexors and extensors; the average peak eccentric moment is higher, with a $26.1 \%$ difference in the flexors and $24.65 \%$ in the extensors, when all the subjects are evaluated together. The data also show strong and significant positive correlations between the PM values for concentric and eccentric of trunk flexors and extensors (Table 4). Thus, concentric and eccentric PM values are directly proportional and related to each other, although they have significantly different mean values, with eccentric values always being higher. Therefore, these variables maintain a greater or directly proportional decrease, showing a significant difference between concentric and eccentric values (figure 2). These results are in accordance with those observed by Helfman (1966), which showed that peak moment production decreases from eccentric to concentric contraction.

Regarding the muscle agonist/antagonist ratio, or trunk extensors/flexors, it is known that an imbalance in muscle strength, with decreased extensor muscle strength relative to flexor muscles, may be a risk factor for lumbar pain (Lee et al., 1999). The literature shows that the relationship between flexor/extensor muscle trunk PM remains between 0.71 and 0.92 for healthy individuals (Delitto et al., 1991; Gómez et al., 2005; Yahia et al., 2010). This is in accordance with the data shown in Table 3 , in which the mean value for men is 0.81 and for women is 0.92 .

There is a variation of the trunk extensor/flexor relationship between the present study and the studies by Delitto et al. (1991) and Gómez et al. (2015), with the present study having the highest values of the aforementioned relation. Although the three studies presented values among the most commonly found in the literature (0.71 and 0.92).

Regarding angular momentum, the PM of the flexor muscles in concentric contraction occurred at an average angle of $56.32^{\circ}$ of trunk flexion, and peak of the extensor muscles at an average of $39.90^{\circ}$ of trunk flexion. In eccentric contraction, the PM of the flexor muscles occurred at an average angle of $53.06^{\circ}$ of trunk flexion, and PM of the extensor muscles at an angle of $33.92^{\circ}$ of trunk flexion, evidencing angular moments very similar in concentric and eccentric contraction. These results are similar to those found by Gómez et al. (2005), which were obtained for the same angular velocity and range of motion in concentric contraction. However, we did not find studies that evaluated the angular momentum of $\mathrm{PM}$ in eccentric contraction.

By determining the isokinetic PM angle, it is possible to establish criteria for movement execution to strengthen specific trunk muscle groups, thus optimizing muscle rehabilitation of the analysed segment. 


\section{CONCLUSION}

The current study indicates that the concentric and eccentric peak moment values of the flexor and extensor trunk muscles are correlated and directly proportional to each other, despite presenting significantly different mean values. The study also shows notably higher peak moment values for men when compared to women for both flexor and extensor muscles in concentric and eccentric contraction.

The results of the present study reflect in part the findings of others on the same topic. The authors believe that due to the incipience of studies in this topic and specifically with these variables, these findings should be considered mainly regarding eccentric values and to peak moment angle if not yet extensively investigated in other studies. The authors also believe that on account of the characteristics of the study population, the peak moment values found could serve as a minimum acceptable standard of trunk muscle strength and balance. Moreover, they could be used as a parameter for diagnosis and comparison for studying symptomatic patients, athletes and other populations with specific characteristics. Furthermore, defining the peak moment angle for the trunk movement range enables the establishment of criteria for the execution of strengthening movements of specific trunk muscle groups, thereby optimizing muscle rehabilitation of the analysed segment.

\section{Acknowledgments: \\ Coordination for the Improvement of Higher Education Personnel (CAPES) granted a scholarship to first author}

Conflict of interests:

Nothing to declare.

Funding:

Nothing to declare.

\section{REFERENCES}

Barczyk-Pawelec, K., Piechura, J. R., Dziubek, W., \& Rozek, K. (2015). Evaluation of isokinetic trunk muscle strength in adolescents with normal and abnormal postures. Journal of Manipulative and
Physiological Therapeutics, 38(7), 484-492. doi:10.1016/j.jmpt.2015.06.010

Cho, K. H., Beom, J. W., Lee, T. S., Lim, J. H., Lee, T. H., \& Yuk, J. H. (2014). Trunk Muscles Strength as a Risk Factor for Nonspecific Low Back Pain: A Pilot Study. Annals of Rehabilitation Medicine, 38(2), 234-240. doi:10.5535/arm.2014.38.2.234

CYBEX (1995). CYBEX NORM ${ }^{\mathrm{TM}}$ testing \& rehabilitation system. Service \& Parts Manual.

Delitto, A., Rose, S. J., Crandell, C. E., \& Strube, M. J. (1991). Reliability of isokinetic measurements of trunk muscle performance. Spine, 16(7), 800-803.

den Hartog, D., Eker, H. H., Tuinebreijer, W. E., Kleinrensink, G. J., Stam, H. J., \& Lange, J. F. (2010). Isokinetic strength of the trunk flexor muscles after surgical repair for incisional hernia. Hernia, 14(3), 243-247. doi:10.1007/s10029010-0627-6

Gómez, H. B., Zarco, R. C., Arias, D. C., García, M. P. D., Hernández, S. R. L., \& Martinez, E. (2005). Valaración isocinética del tronco em sujetos asintomáticos del Centro Nacional de Rehabilitación. Acta Ortopédica Mexicana, 19, 4955.

Grabiner, M. D., Jeziorowski, J. J., \& Divekar, A. D. (1990). Isokinetic measurements of trunk extension and flexion performance collected with the biodex clinical data station. Journal of Orthopaedic \& Sports Physical Therapy, 11 (12), 590598.

Guilhem, G., Giroux, C., Couturier, A., \& Maffiuletti, N. A. (2014). Validity of trunk extensor and flexor torque measurements using isokinetic dynamometry. Journal of Electromyography and Kinesiology, 24(6), 986-993. doi:10.1016/j.jelekin.2014.07.006

Haight, H. J., Dahm, D. L., Smith, J., \& Krause, D. A. (2005). Measuring standing hindfoot alignment: reliability of goniometric and visual measurements. Archives of Physical Medicine and Rehabilitation, 86(3), 571-575. doi:10.1016/j.apmr.2004.05.014

Helfman, H. (1966). Biomechanics of muscle. Journal of Bone and Joint Surgery, 44(A), 363-373.

Hides, J., Stanton, W., Mendis, M. D., \& Sexton, M. (2011). The relationship of transversus abdominis and lumbar multifidus clinical muscle tests in patients with chronic low back pain. Manual Therapy, 16(6), 573-577. doi:10.1016/j.math.2011.05.007

Hupli, M., Sainio, P., Hurri, H., \& Alaranta, H. (1997). Comparison of trunk strength measurements between two different isokinetic devices used at clinical settings. Journal of Spinal Disorders, 10(5), 391-397.

Jeon, K., \& Kim, S. (2016). Effect of unilateral exercise on spinal and pelvic deformities, and isokinetic trunk muscle strength. Journal of Physical Therapy Science, 28(3), 844-849. doi:10.1589/jpts.28.844

Lee, J., Hoshino, Y., Nakamura, K., Kariya, Y., Saita, K., \& Ito, K. (1999). Trunk Muscle Weakness as a Risk Factor for Low Back Pain: A 5-Year 
Prospective Study. Spine, 24(1), 54-57. doi:10.1097/00007632-199901010-00013

Lim, E. C., Poh, R. L., Low, A. Y., \& Wong, W. P. (2011). Effects of Pilates-based exercises on pain and disability in individuals with persistent nonspecific low back pain: a systematic review with meta-analysis. Journal of Orthopaedic \& Sports Physical Therapy, 41(2), 70-80. doi:10.2519/jospt.2011.3393

Marshall, P. W., Kennedy, S., Brooks, C., \& Lonsdale, C. (2013). Pilates exercise or stationary cycling for chronic nonspecific low back pain: does it matter? a randomized controlled trial with 6month follow-up. Spine, 38(15), 952-959. doi:10.1097/BRS.0b013e318297c1e5.

Mostagi, F. Q., Dias, J. M., Pereira, L. M., Obara, K., Mazuquin, B. F., Silva, M. F., . . . Cardoso, J. R. (2015). Pilates versus general exercise effectiveness on pain and functionality in nonspecific chronic low back pain subjects. Journal of Bodywork and Movement Therapies, 19(4), 636-645. doi:10.1016/j.jbmt.2014.11.009

Newton, M., \& Waddell, G. (1993). Trunk strength testing with iso-machines. Part 1: Review of a decade of scientific evidence. Spine, 18(7), 801811.

Pereira, L. M., Obara, K., Dias, J. M., Menacho, M. O., Guariglia, D. A., Schiavoni, D., . . Cardoso, J. R. (2012). Comparing the Pilates method with no exercise or lumbar stabilization for pain and functionality in patients with chronic low back pain: systematic review and meta-analysis. Clinical Rehabilitation, 26(1), 10-20. doi:10.1177/0269215511411113

Pinto, R. Z., Souza, T. R., Trede, R. G., Kirkwood, R. N., Figueiredo, E. M., \& Fonseca, S. T. (2008). Bilateral and unilateral increases in calcaneal eversion affect pelvic alignment in standing position. Manual Therapy, 13(6), 513-519. doi:10.1016/j.math.2007.06.004

Silva Neto, M., Simões, R., Grangeiro Neto, J. A., \& Cardone, C. P. (2010). Avaliação isocinética da força muscular em atletas profissionais de futebol feminino. Revista Brasileira de Medicina do Esporte, 16, 33-35.

Van Damme, B. B., Stevens, V. K., Van Tiggelen, D. E., Duvigneaud, N. N., Neyens, E., \& Danneels, L. A.
(2013). Velocity of isokinetic trunk exercises influences back muscle recruitment patterns in healthy subjects. Journal of Electromyography and Kinesiology, 23(2), 378-386. doi:10.1016/j.jelekin.2012.10.015

van Middelkoop, M., Rubinstein, S. M., Kuijpers, T., Verhagen, A. P., Ostelo, R., Koes, B. W., \& van Tulder, M. W. (2011). A systematic review on the effectiveness of physical and rehabilitation interventions for chronic non-specific low back pain. European Spine Journal, 20(1), 19-39. doi:10.1007/s00586-010-1518-3

van Middelkoop, M., Rubinstein, S. M., Verhagen, A. P., Ostelo, R. W., Koes, B. W., \& van Tulder, M. W. (2010). Exercise therapy for chronic nonspecific low-back pain. Best Practice $\mathcal{E}$ Research: Clinical Rheumatology, 24(2), 193-204. doi:10.1016/j.berh.2010.01.002

Wajswelner, H., Metcalf, B., \& Bennell, K. (2012). Clinical pilates versus general exercise for chronic low back pain: randomized trial. Medicine $\mathcal{E}$ Science in Sports \& Exercise, 44(7), 1197-1205. doi:10.1249/MSS.0b013e318248f665

Wells, C., Kolt, G. S., Marshall, P., Hill, B., \& Bialocerkowski, A. (2013). Effectiveness of Pilates exercise in treating people with chronic low back pain: a systematic review of systematic reviews. BMC Medical Research Methodology, 13, 7. doi:10.1186/1471-2288-13-7

WHO (2010). Global recommendations on physical activity for health. World Health Organization.

WHO (2013). Department of Chronic Diseases and Health Promotion and Chronic Respiratory Diseases and Arthritis. World Health Organization.

Yahia, A., Ghroubi, S., Kharrat, O., Jribi, S., Elleuch, M., \& Elleuch, M. H. (2010). A study of isokinetic trunk and knee muscle strength in patients with chronic sciatica. Annals of Physical and Rehabilitation Medicine, 53(4), 239-244, 244-239. doi:10.1016/j.rehab.2010.03.002

Yahia, A., Jribi, S., Ghroubi, S., Elleuch, M., Baklouti, S., \& Habib Elleuch, M. (2011). Evaluation of the posture and muscular strength of the trunk and inferior members of patients with chronic lumbar pain. Joint Bone Spine, 78(3), 291-297. doi:10.1016/j.jbspin.2010.09.008 